\title{
The impact of driver sleepiness on fixation- related brain potentials
}

Christer Ahlström, Ignacio Solis-Marcos, Emma Nilsson and Torbjorn Akerstedt

The self-archived postprint version of this journal article is available at Linköping University Institutional Repository (DiVA):

http://urn.kb.se/resolve?urn=urn:nbn:se:liu:diva-162944

N.B.: When citing this work, cite the original publication.

Ahlström, C., Solis-Marcos, I., Nilsson, E., Akerstedt, T., (2020), The impact of driver sleepiness on fixation-related brain potentials, Journal of Sleep Research, 29(5), e12962.

https://doi.org/10.1111/jsr.12962

Original publication available at:

https://doi.org/10.1111/jsr.12962

Copyright: Wiley

https://www.wiley.com/en-gb 
Title: The impact of driver sleepiness on fixation-related brain potentials

Short title: Lambda response and driver sleepiness

Authors: Christer Ahlström ${ }^{1,2}$, Ignacio Solis-Marcos ${ }^{1}$, Emma Nilsson $^{3,4}$, Torbjörn Åkerstedt ${ }^{5,6}$

${ }^{1}$ Swedish National Road and Transport Research Institute (VTI), Linköping, Sweden

${ }^{2}$ Department of Biomedical Engineering, Linköping University, Linköping, Sweden

${ }^{3}$ Volvo Cars Safety Centre, Volvo Car Corporation, Göteborg, Sweden

${ }^{4}$ Department of Mechanics and Maritime Sciences, Chalmers University of Technology, Göteborg, Sweden

${ }^{5}$ Stress Research Institute, Stockholm University, Stockholm, Sweden

${ }^{6}$ Clinical Neuroscience, Karolinska Institute, Stockholm, Sweden

\section{Corresponding author:}

Christer Ahlström

VTI

S-58195 Linköping

Sweden

Phone: +46 13204009

Fax: +4613141436

Email: christer.ahlstrom@vti.se 


\begin{abstract}
The effects of driver sleepiness are often quantified as deteriorated driving performance, increased blink durations, and high levels of subjective sleepiness. Driver sleepiness has also been associated with increasing levels of EEG power, especially in the alpha range. The present exploratory study investigated a new measure of driver sleepiness, the EEG fixation-related lambda response. Thirty young male drivers $(23.6 \pm 1.7$ years old $)$ participated in a driving simulator experiment in which they drove on rural and suburban roads in simulated daylight versus darkness during both daytime (full sleep) and night-time (sleep deprived). The results show lower lambda responses during night driving and with longer time on task, indicating that sleep deprivation and time on task cause a general decrement in cortical responsiveness to incoming visual stimuli. Levels of subjective sleepiness and line crossings were higher under the same conditions. Furthermore, results of a linear mixed-effects model showed that low lambda responses are associated with high subjective sleepiness and more line crossings. We suggest that the fixation-related lambda response can be used to investigate driving impairment induced by sleep deprivation while driving and that, after further refinement, it may be useful as an objective measure of driver sleepiness.
\end{abstract}

Keywords: driver sleepiness, event-related potential, fixation-related ERP, P1, line crossings

\title{
1 Introduction
}

Sleepy drivers are usually able to sustain performance until they eventually falter and momentarily fall asleep. This wake-state instability, in which the sleep-deprived brain can switch back and forth between alert and asleep in a matter of seconds, is deceptive (Krause et al., 2017). Not only does it make it difficult to predict when an episode of microsleep will occur, it may also, via local sleep, lead to performance failures in seemingly awake individuals (Ahlstrom et al., 2017, Nir et al., 2017, Quercia et al., 2018). This is accompanied by a slowing of visual processing, loss of selective attention, distractor inhibition, and reduced peripheral processing capacity (Chee, 2015). In this paper, this degradation in visual information processing is investigated using EEG event-related potentials (ERPs), time-locked to fixation onsets, and their relationship to driver sleepiness, sleep deprivation, and time on task.

ERPs are associated with attention and working memory tasks and allow insights into cortical processing. The so-called N1 component (a negative wave approximately $100 \mathrm{~ms}$ after the stimulus event) is associated with the processing of auditory, visual, or tactile stimuli in primary sensory areas (Rennie et al., 2002) and is modulated by attention and conscious visual perception (Luck et al., 2000, Railo et al., 2011). P1 (a positive wave approximately $100 \mathrm{~ms}$ after the stimulus) is also modulated by 
attention and reflects the processing of visual stimuli (Luck et al., 2000). P3 (a positive wave approximately $300 \mathrm{~ms}$ after the stimulus) is thought to reflect higher cognitive information processing such as memory, attention, and executive function (van Dinteren et al., 2014). Interestingly, the amplitudes of all these ERP components have been found to decrease with sleep deprivation (Gosselin et al., 2005, Hoedlmoser et al., 2011). This suggests that sleep deprivation causes a general decrement in information processing, attention, and cortical responsiveness to incoming stimuli (Boonstra et al., 2007, Chee, 2015).

ERPs are usually calculated by averaging EEG traces time-locked to repeated artificial sensory, motor, or cognitive events such as auditory tones or blinking lights. Examples of such artificially induced triggers used in a driving setting are auditory or visual oddball tasks (Solís-Marcos et al., 2017, Strayer et al., 2015). However, in real-life driving, these artificial stimuli are not appropriate as they are obtrusive, interfere with the driving task, and may change the character of the experimental situation. In a driver sleepiness setting, artificial stimuli may also interfere with the process of falling asleep. A solution to these problems may be to use naturally occurring events, such as saccades or eye blinks, as triggers (Takeda et al., 2012). Using oculomotor events as triggers is guided by the notions that eye blinks cluster around certain moments in information processing (Berg and Davies, 1988, Wascher et al., 2014), and that the onset of fixations demarcate the inflow of new visual information (Yagi, 1979). Eye fixation-related ERPs (EFRPs) have been shown to be related to the planning, programming, generation, and execution of saccadic eye movements, and to the encoding of new visual information following a saccade (Jagla et al., 2007, Nikolaev et al., 2016). The most prominent EFRP is a positive wave 80-100 ms after fixation onset called the lambda response (Jagla et al., 2007, Kazai and Yagi, 2003, Nikolaev et al., 2016). The lambda response has been as a correspondence to P1 in visually triggered ERPs, with a common neurological origin in the visual cortex (Kazai and Yagi, 2003). Its amplitude is influenced by attention, interest, and distraction (Yagi, 1981, Ries et al., 2016), and in driving studies, the lambda response has been shown to increase with increased driving demand (Wiberg et al., 2015) and decrease with increased cognitive distraction (Takeda et al., 2012).

Given that the lambda response is related to visual information processing depth (Takeda et al., 2016, Takeda et al., 2012, Yagi, 1981, Jagla et al., 2007), and that artificially triggered P1 amplitudes decrease with sleep deprivation (Hoedlmoser et al., 2011), we hypothesize that the lambda response will decrease with sleep deprivation and time on task. This would indicate that insufficient sleep causes reduced attention to visual sensory input while driving. We also hypothesize that the lambda response will be related to subjective sleepiness and involuntary line crossings. The main purpose of this exploratory study was to investigate the effect of night driving on the lambda response. A secondary purpose was to relate the lambda response to two established sleepiness indicators, subjective sleepiness and line crossings. 


\section{Materials and methods}

\subsection{Participants}

Thirty participants (age $23.6 \pm 1.7$ years) were randomly selected from the Swedish register of vehicle owners. Young drivers (18-25 years old) were chosen because they are overrepresented in sleeprelated crashes (Filtness et al., 2012). To minimize the potential confounds of menstrual cycles or contraceptive use, only males were selected. Only individuals with relatively normal sleep patterns were included, that is, individuals with day work, without any sleep disorder, and with normal BMI < 30 (to reduce the risk of sleepiness due to obstructive sleep disorders). Distinctive morning types were excluded to avoid high levels of sleepiness in the daytime condition, which extended into the evening hours. The study was approved by the local ethics committee in Linköping (Dnr 2014/309-31).

\subsection{Design and procedure}

The study took place in an advanced moving-base driving simulator (VTI Sim III) at the Swedish National Road and Transport Research Institute (VTI). The simulator consists of a car cabin positioned on a motion platform that offers both linear and tilting motion. The graphics are provided by six SXRD projectors giving a 120-degree forward field of vision and three LCD displays representing the rear-view mirrors.

The participants visited the laboratory on six separate occasions, three times during the daytime and three times during the night-time. The daytime sessions (full sleep) were run between $12.30 \mathrm{~h}$ and $21.15 \mathrm{~h}$ and the night sessions (sleep deprived) were run between $22.00 \mathrm{~h}$ and $06.15 \mathrm{~h}$. Participants were instructed to sleep for at least $7 \mathrm{~h}$ on each of the three days before the trials, to go to bed no later than $24.00 \mathrm{~h}$, and to get up no later than $09.00 \mathrm{~h}$. They were also requested to avoid alcohol for $72 \mathrm{~h}$ and to abstain from nicotine and caffeine for $3 \mathrm{~h}$ before driving. Upon arrival at the laboratory, the participants were informed about the study and signed an informed-consent form. After that, electrodes were mounted for the physiological measurements.

The experiment was designed to investigate driver sleepiness in relation to light condition (daylight/darkness), traffic environment (rural/suburban), and consistency (three identical repetitions for each driver). Note that driving in both daylight and darkness was experienced during both daytime and night-time. The participants drove three simulated scenarios on each of their six visits: (i) a rural road with a speed limit of $80 \mathrm{~km} / \mathrm{h}$ in daylight, (ii) the same rural road in darkness, and (iii) a suburban road in daylight with high traffic density and a speed limit of $60 \mathrm{~km} / \mathrm{h}$. Each scenario lasted $30 \mathrm{~min}$, and there were $1.5 \mathrm{~h}$ of rest between each drive. The order of the three scenarios was randomized between participants and the daytime/night-time conditions were balanced. Results concerning light condition (scenarios i and ii) are reported in Ahlström et al. (2018a), whereas results concerning the driving environment (scenarios i and iii) are reported in Ahlström et al. (2018b). 
Subjective sleepiness was rated every fifth minute using the 9-point Karolinska Sleepiness Scale (KSS) (Åkerstedt et al., 2014).

\subsection{Data and pre-processing}

An electrooculogram (EOG) and a 30-channel electroencephalogram (EEG) were recorded using a multi-channel amplifier with active electrodes (g.HIamp, g.tec Medical Engineering GmbH, Austria). The EEG electrodes were positioned according to the 10-20 system and the EOG electrodes were positioned horizontally and vertically across the left eye. The right earlobe was used as a reference. The physiological signals were digitized at $256 \mathrm{~Hz}$, band-pass filtered between 0.5 and $60 \mathrm{~Hz}$ using an $8^{\text {th }}$-order Butterworth filter, and notch filtered to remove $50 \mathrm{~Hz}$ line noise. Impedance levels were checked before the experiment and adjusted after each condition as needed. Segments with large motion artefacts in the EEG were manually rejected. This was followed by automatic removal of eye movement artefacts, based on independent component analysis, according to Daly et al. (2015). Saccades were extracted from the horizontal EOG using a wavelet-based approach suggested by Bulling et al. (2011). Physiologically unlikely saccade candidates were removed if they had a saccade amplitude below $20 \mu \mathrm{V}$ or a saccade duration $<10 \mathrm{~ms}$ or $>150 \mathrm{~ms}$.

EFRPs were obtained by averaging EEG data time-locked to the fixation onset according to Takeda et al. $(2016,2012)$, with the fixation onset $\left(t_{\text {fixation }}\right)$ defined as the first local extrema succeeding the point of maximum velocity of saccadic eye movements. Fixation onsets that co-occurred with eye blinks (detected according to Jammes et al., 2008) were excluded from the analysis to reduce the influence of oculomotor activity on the EFRPs. Baseline correction was applied for an interval between 300 and $150 \mathrm{~ms}$ preceding $t_{\text {fixation }}$ to avoid the inclusion of saccade-related activity in the baseline calculation. EFRPs were calculated at nine parieto-occipital electrode sites (i.e. P3, Pz, P4, POz, O1, Oz, and O2) where the EFRP P1 component is most evident (Kazai and Yagi, 2003). Ensemble averaging of EEG data was carried out across fixation onsets in 5-min segments corresponding to the KSS ratings. The number of epochs that went into the ensemble averages was in the range of $18-326\left(1^{\text {st }}\right.$ quartile $=45$, median $=110$, and $3^{\text {rd }}$ quartile $=158$ ). A trimmed mean with $10 \%$ rejection was used for ensemble averaging to reduce the impact of outliers and noise. The lambda response was extracted as the largest local maximum in a time window from 20 to $100 \mathrm{~ms}$ after $t_{\text {fixation }}$ in the POz electrode.

In total, 540 (30 drivers $\times 6$ occasions $\times 3$ drives) driving sessions were conducted. Data from the first daytime visit and first night-time visit were excluded to eliminate first encounter effects. Also, four participants were excluded since they failed to comply with the requirements on prior sleep. This resulted in 312 driving sessions. From these, 1639 5-min segments (87.5\%) were considered to have sufficient signal quality in the horizontal EOG and the POz channel and were selected for the final analyses. 


\subsection{Statistical analysis}

The effects on lambda response, subjective sleepiness ( $\mathrm{KSS} \leq 5, \mathrm{KSS}=6, \mathrm{KSS}=7, \mathrm{KSS}=8$, and $\mathrm{KSS}$ $=9)$, and number of line crossings per $5 \min (0,1,2,3, \geq 4)$ were analysed with mixed-model analyses of variance (ANOVA) using the fixed factors daytime condition (day - normal sleep versus night sleep deprived) and time on task (the six 5-min segments in each drive) as the dependent variables. Participant (1-26) was used as a random factor. The amplitude of the saccade preceding the fixation onset (small saccades $<100 \mathrm{~ms}$ versus large saccades $\geq 100 \mathrm{~ms}$ ), number of fixation onsets (grouped with a class interval of 100 fixations), light condition (simulated daylight versus simulated darkness), and driving environment (rural versus suburban) were included as confounding factors, since lambda responses depend on eye movements and saccade size (Nikolaev et al., 2016, Takeda et al., 2016) and the driving scenario variables affect sleepiness in the present dataset (Ahlström et al., 2018b, Ahlström et al., 2018a). The interaction between condition and time on task was also included in the model. Post hoc tests with pairwise comparisons were performed using Tukey's honest significant difference criterion with the alpha level set to 0.05 .

A linear mixed-effects model was used to analyse how well the lambda response predicts subjective sleepiness and line crossings, respectively. Lambda response, light condition, driving environment, saccade amplitude, and number of fixations were used as predictor variables in the model, and participant was included as a random effect. Separate analyses were performed for subjective sleepiness and line crossings. Reported results are based on maximum likelihood estimation and Cholesky parameterization was used to estimate the covariance structure.

To provide a reference for the previous analyses, two additional ANOVAs were carried out using subjective sleepiness as the main dependent variable, saccade amplitude, number of fixations, light condition, and road environment as confounding factors, participant as a random factor, and lambda response and line crossings as the independent variables. Post hoc tests were performed as described above.

All analyses were carried out in Matlab 9.5 using the Statistics and Machine Learning Toolbox 11.4 (Mathworks Inc., MA, USA). The anovan function was used for the ANOVAs and fitlme was used for the linear mixed-effects models.

\section{Results}

Night driving as well as higher ratings of subjective sleepiness were associated with lower lambda responses (Figure 1 and Figure 2). The highest lambda response was observed at the POz electrode with a peak approximately $55 \mathrm{~ms}$ after $t_{\text {fixation. }}$. The results display a dose-response pattern, with the highest amplitudes for KSS $\leq 5$ and the lowest values for $\mathrm{KSS}=9$. 

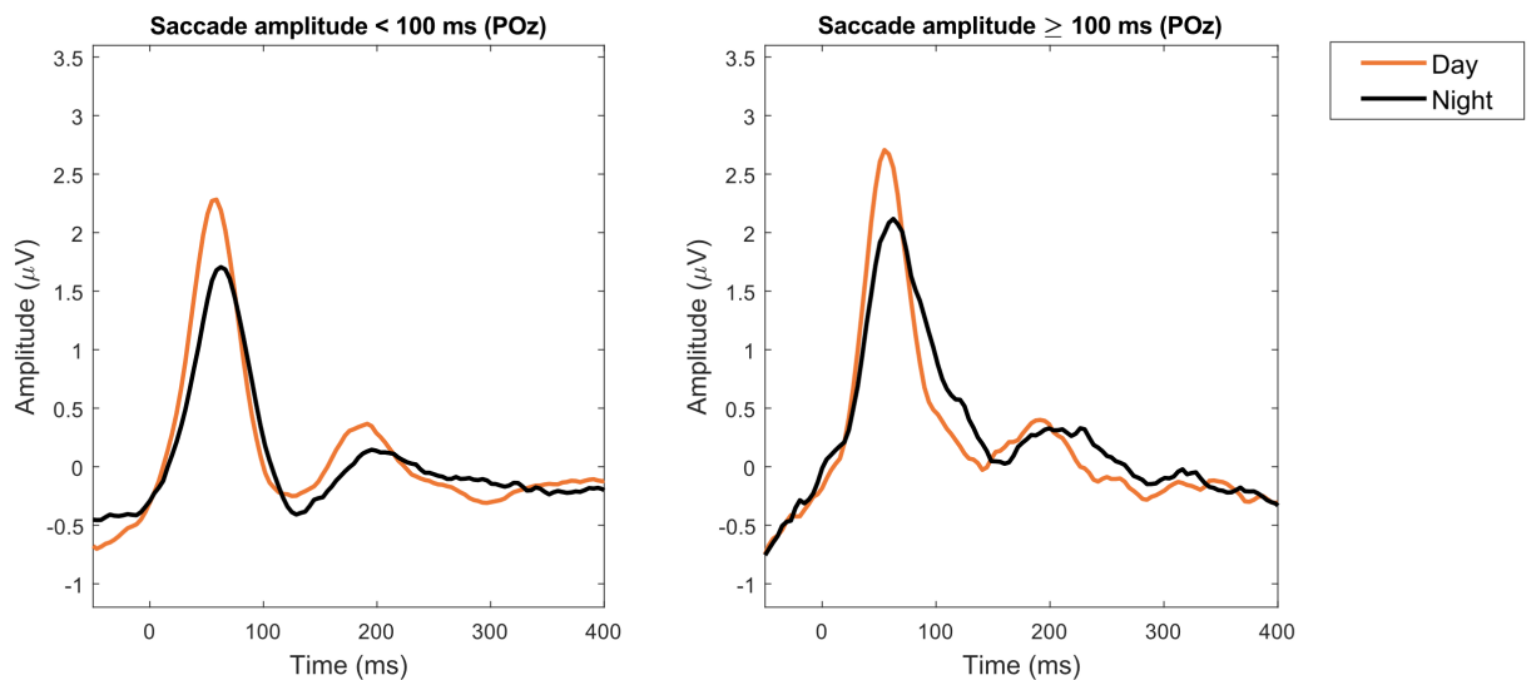

Figure 1 Grand average EFRPs from the POz electrode. The two curves represent EFRPs for the daytime condition (day/night), separated between small and large saccades.
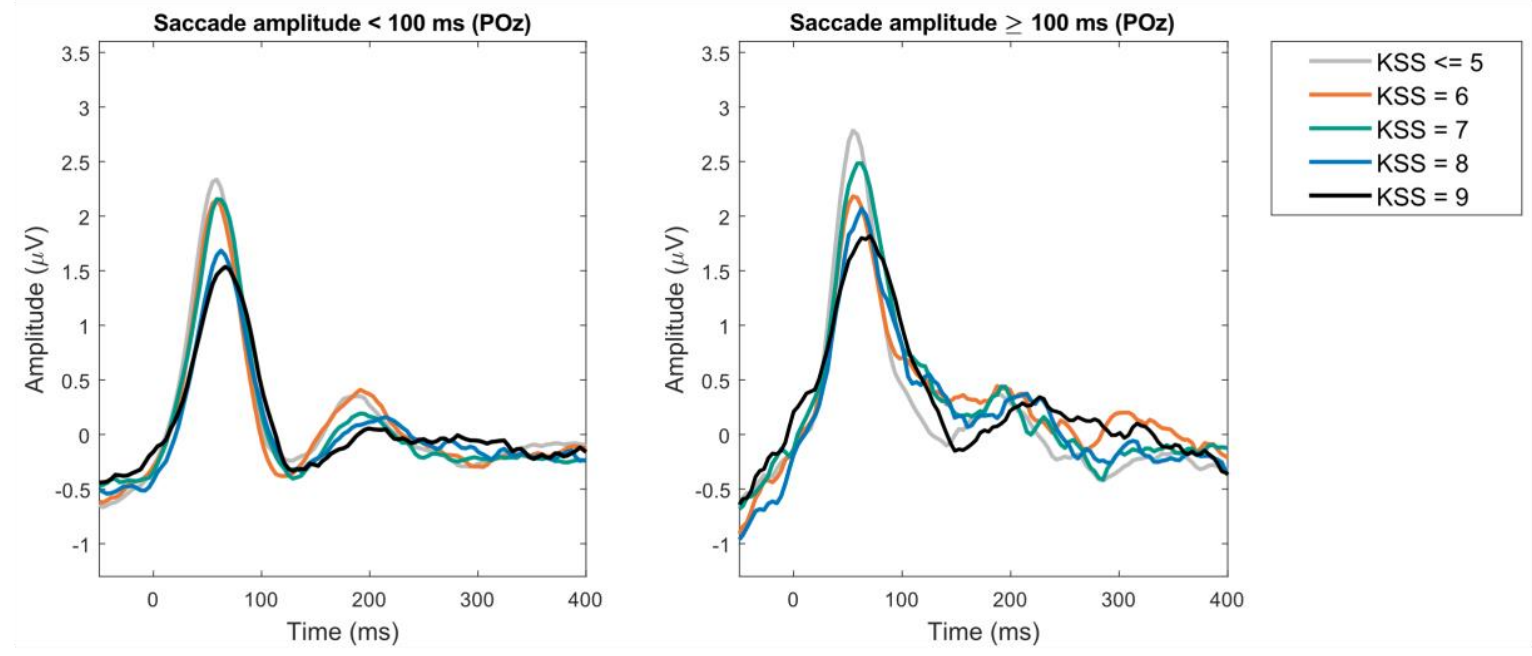

Figure 2 Grand average EFRPs from the POz electrode. The five curves represent EFRPs for subjective sleepiness according to $\mathrm{KSS} \leq 5, \mathrm{KSS}=6, \mathrm{KSS}=7, \mathrm{KSS}=8$, and $\mathrm{KSS}=9$, separated between small and large saccades.

The lambda response decreased during night driving (see Table 1 and Figure 3). It also decreased with time on task, with significantly lower amplitudes after the first $10 \mathrm{~min}$ of driving. In addition, the lambda response decreased when driving in suburban versus rural environments, and when the lambda response was time-locked to small saccades. As expected, subjective sleepiness and line crossings increased with sleep deprivation and time on task. Interaction effects between sleep deprivation and time on task were found for subjective sleepiness and line crossings, with a more rapid increase in KSS ratings and line crossings after sleep deprivation. 
Table 1 F-values, degrees of freedom, and effect sizes (partial $\eta^{2}$ ) from mixed-model ANOVAs for daytime condition (day/night) and time on task. Light condition, driving environment, and saccade amplitude are considered confounding variables and participant is included as a random factor. Significant differences at the 0.05 level are indicated with one star and at the $<0.001$ level with two stars.

\begin{tabular}{|c|c|c|c|}
\hline & Lambda response & Subjective sleepiness & Line crossings \\
\hline Daytime (day/night) & $\begin{array}{c}\mathrm{F}_{(1,3271)}=40.8 * *, \\
\eta^{2}=0.01\end{array}$ & $\begin{array}{c}\mathrm{F}_{(1,1597)}=1051.8 * * \\
\eta^{2}=0.40\end{array}$ & $\begin{array}{c}\mathrm{F}_{(1,1599)}=112.7 * *, \\
\eta^{2}=0.07\end{array}$ \\
\hline Time on task & $\begin{array}{c}\mathrm{F}_{(5,3271)}=8.9 * * \\
\eta^{2}=0.01\end{array}$ & $\begin{array}{c}\mathrm{F}_{(5,1597)}=38.9 * *, \\
\eta^{2}=0.11\end{array}$ & $\begin{array}{c}\mathrm{F}_{(5,1599)}=13.4 * *, \\
\eta^{2}=0.04\end{array}$ \\
\hline $\begin{array}{l}\text { Saccade amplitude } \\
\text { (small/large) }\end{array}$ & $\begin{array}{c}\mathrm{F}_{(1,3271)}=51.8 * *, \\
\eta^{2}=0.02\end{array}$ & - & - \\
\hline Number of fixations & $\begin{array}{c}\mathrm{F}_{(5,3271)}=1.5 \\
\eta^{2}=0.002\end{array}$ & $\begin{array}{c}\mathrm{F}_{(5,1597)}=29.0 * *, \\
\eta^{2}=0.03\end{array}$ & $\begin{array}{c}\mathrm{F}_{(5,1599)}=4.1^{*} \\
\eta^{2}=0.005\end{array}$ \\
\hline $\begin{array}{l}\text { Light } \\
\text { (daylight/darkness) }\end{array}$ & $\begin{array}{c}\mathrm{F}_{(1,3271)}=1.8 \\
\eta^{2}=0.001\end{array}$ & $\begin{array}{c}\mathrm{F}_{(1,1597)}=7.9 * * \\
\eta^{2}=0.005\end{array}$ & $\begin{array}{c}\mathrm{F}_{(1,1599)}=0.1 \\
\eta^{2}=0\end{array}$ \\
\hline $\begin{array}{l}\text { Environment } \\
\text { (rural/suburban) }\end{array}$ & $\begin{array}{c}\mathrm{F}_{(1,3271)}=10.4^{* *}, \\
\eta^{2}=0.003\end{array}$ & $\begin{array}{c}\mathrm{F}_{(1,1597)}=15.7 * *, \\
\eta^{2}=0.01\end{array}$ & $\begin{array}{c}\mathrm{F}_{(1,1599)}=2.2 \\
\eta^{2}=0.001\end{array}$ \\
\hline Participant & $\begin{array}{c}\mathrm{F}_{(24,3271)}=53.4 * *, \\
\eta^{2}=0.28\end{array}$ & $\begin{array}{c}\mathrm{F}_{(24,1597)}=40.6^{* *}, \\
\eta^{2}=0.38\end{array}$ & $\begin{array}{c}\mathrm{F}_{(24,1599)}=26.9 * *, \\
\eta^{2}=0.29\end{array}$ \\
\hline $\begin{array}{l}\text { Daytime } * \text { Time on } \\
\text { task }\end{array}$ & $\begin{array}{c}\mathrm{F}_{(5,3271)}=0.5 \\
\eta^{2}=0.001\end{array}$ & $\begin{array}{c}\mathrm{F}_{(5,1597)}=2.53^{*}, \\
\eta^{2}=0.008\end{array}$ & $\begin{array}{c}\mathrm{F}_{(5,1599)}=5.1 * * \\
\eta^{2}=0.02\end{array}$ \\
\hline
\end{tabular}
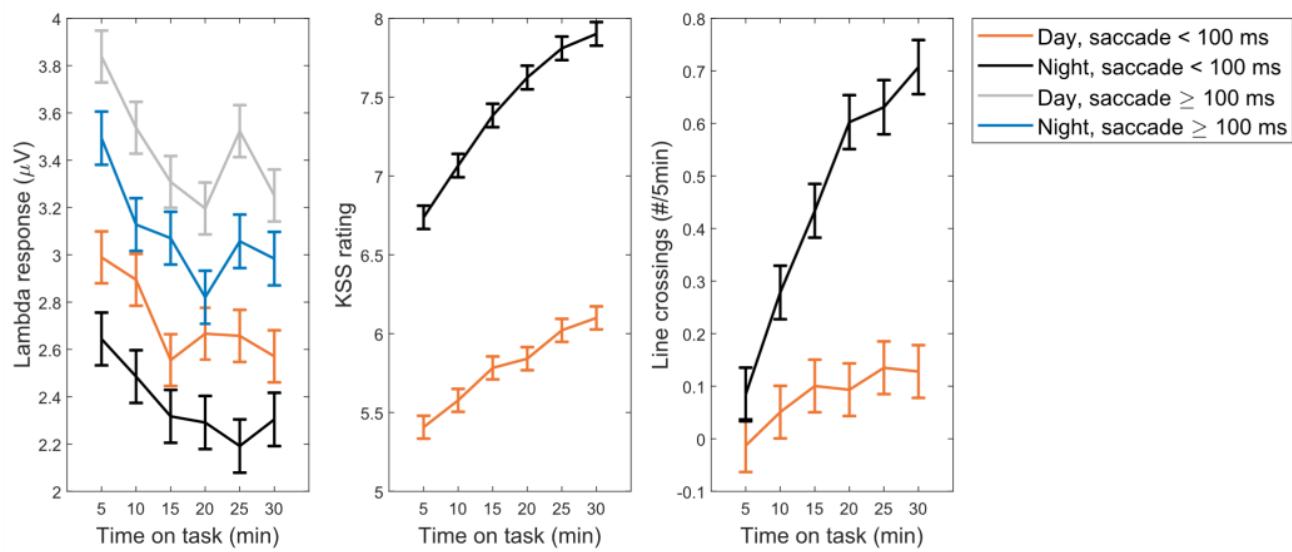

Figure 3 The estimated mean values of lambda responses, KSS, and number of line crossings for each of the six segments (time on task), separated by daytime condition (day/night). The lambda response is also separated between short and long saccades. The error bars represent the standard error of the mean for each condition.

The linear mixed-effects model showed a significant relationship between the lambda response and subjective sleepiness and line crossings when controlling for individual differences. As the lambda response increased by $1 \mu \mathrm{V}$, the KSS level decreased by 0.17 and there were on average 0.04 fewer line crossings per 5 min of driving. Light condition, environment, saccade amplitude, and number of 
fixations were also found to be significant predictors of KSS (Table 2). Figure 4 illustrates the large variation in lambda response between individuals, as well as the rather limited range of responses per individual. The proportion of variability in subjective sleepiness and line crossings explained by the models was $33 \%$ and $26 \%$, respectively.
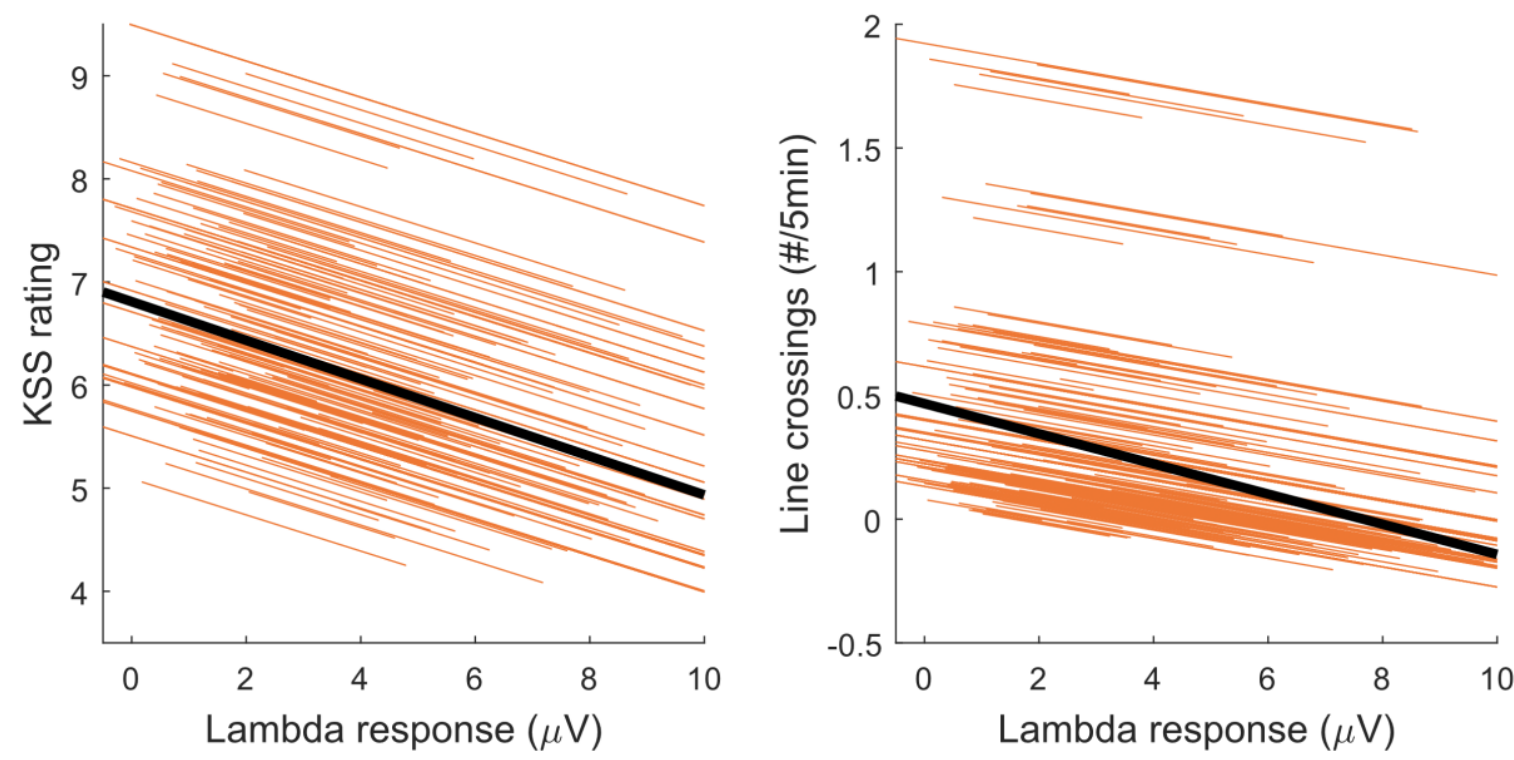

Figure 4 The estimated linear mixed-effects model of subjective sleepiness and line crossings, plotted as a function of lambda response. The grey lines represent the random effects of participants, and the bold red line shows the model without taking participant into account.

Table 2 Linear mixed-effects model of KSS and line crossings using lambda response as a continuous independent variable and light and environment as nominal independent variables. Participant is included as a random factor. The beta coefficients for light, environment, and saccade amplitude are relative values compared with the assumedly most predictable conditions $($ light $=$ daylight, environment $=$ urban $)$.

\section{KSS}

Line crossings

Fixed effects

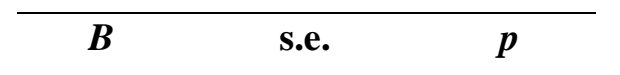

$\begin{array}{lll}B & \text { s.e. } \quad p\end{array}$

\begin{tabular}{rcccccc}
\hline Intercept & 8.80 & 0.23 & $<.0001$ & 0.70 & 0.11 & $<.0001$ \\
Lambda response & -0.17 & 0.01 & $<.0001$ & -0.04 & 0.01 & $<.0001$ \\
SaccadeAmplitude_large & -1.28 & 0.11 & $<.0001$ & -0.21 & 0.03 & 0.05 \\
Number of fixations & -0.01 & $<0.001$ & $<\mathbf{0 . 0 0 1}$ & -0.002 & $<0.001$ & $<\mathbf{0 . 0 0 1}$ \\
Light_night & 0.22 & 0.07 & $\mathbf{0 . 0 0 0 1}$ & -0.03 & 0.03 & 0.36 \\
Environment_rural & -0.36 & 0.07 & $<.0001$ & 0.06 & 0.03 & $\mathbf{0 . 0 4}$ \\
\hline Random effects & $\boldsymbol{B}$ & Lower & $\boldsymbol{U p p e r}$ & $\boldsymbol{B}$ & Lower & $\boldsymbol{U p p e r}$ \\
\hline Intercept (participant) & 0.93 & 0.70 & 1.23 & 0.41 & 0.31 & 0.54
\end{tabular}


To provide a reference for the previous analyses, the relationships between subjective sleepiness and the other variables were investigated. This revealed a relationship between KSS and lambda response for both small and large saccades, with decreasing amplitudes with increasing KSS values (Table 3 and Figure 5). Post hoc analyses showed that lambda responses (small saccade) differed between KSS levels except for the closest neighbouring KSS level (e.g. the lambda response associated with KSS $\leq$ 5 differed from that associated with $\mathrm{KSS} \geq 7$, but not that associated with $\mathrm{KSS}=6$ ). The lambda response was lower when derived from small versus large saccades and when driving on the urban road. Light condition did not affect the lambda response. The number of line crossings increased with increasing levels of subjective sleepiness and were higher on the rural road.
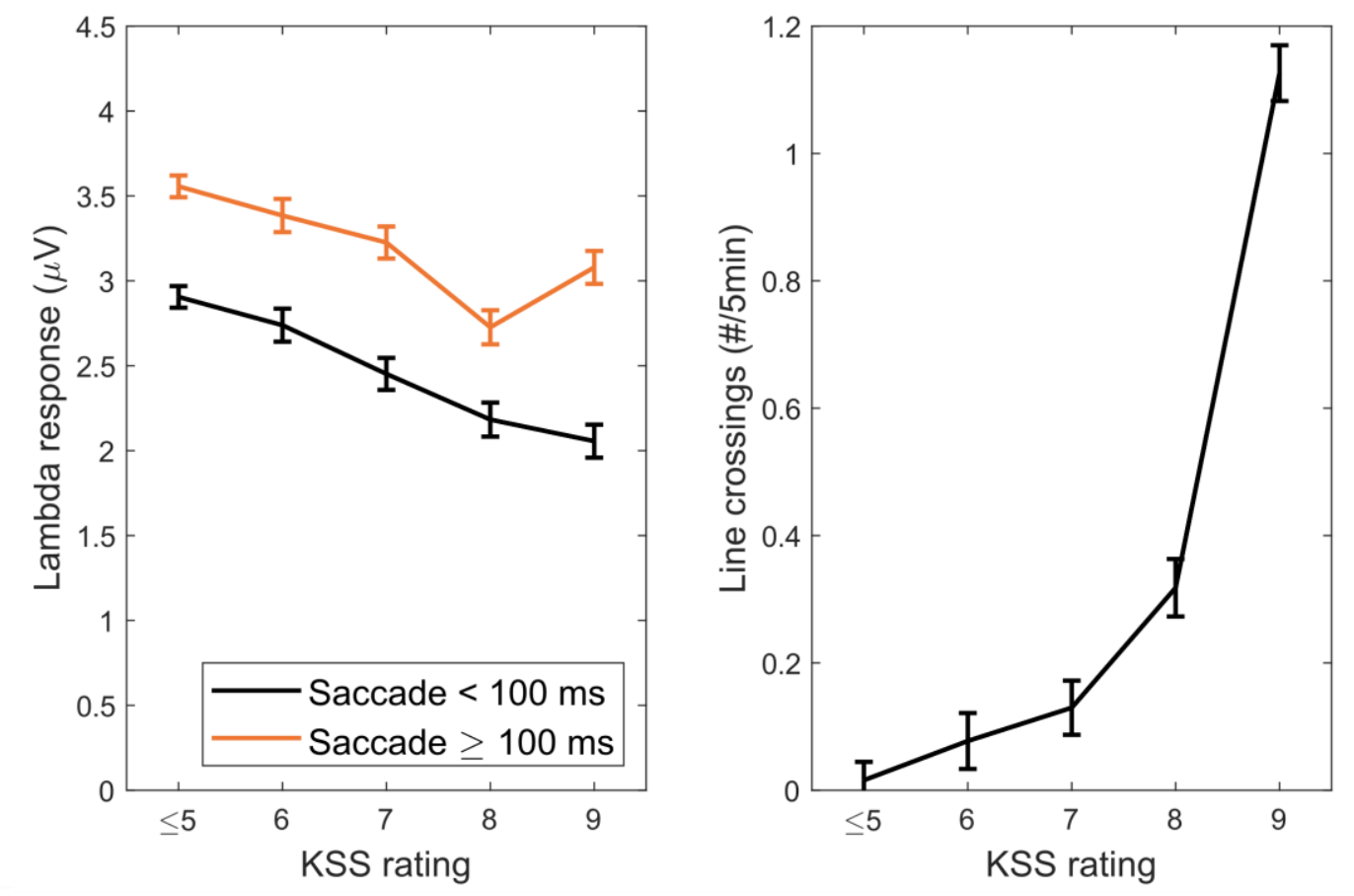

Figure 5 The estimated mean values of the lambda response and number of line crossings as a function of subjective sleepiness. The error bars represent the standard error of the mean for each condition.

Table 3 F-values, degrees of freedom, and effect sizes (partial $\eta^{2}$ ) for subjective sleepiness $(\mathrm{KSS} \leq 5, \mathrm{KSS}=6, \mathrm{KSS}=7$, $\mathrm{KSS}=8$, and KSS =9). Saccade amplitude, light condition, and driving environment are considered confounding variables and participant is included as a random factor. Significant differences at the 0.05 level are indicated with one star and at the $<0.001$ level with two stars.

\begin{tabular}{lcc}
\hline & Lambda response & Line crossings \\
\hline Subjective sleepiness & $\mathrm{F}_{(4,3227)}=26.3^{* *}, \eta^{2}=0.03$ & $\mathrm{~F}_{(4,1601)}=88.2^{* *}, \eta^{2}=0.18$
\end{tabular}


Saccade amplitude (small/large)

Number of fixations

Light (daylight/darkness)

Environment (rural/suburban)

Subjective sleepiness * Saccade amplitude

Participant

$$
\begin{array}{cc}
\mathrm{F}_{(1,3227)}=32.2^{* *}, \eta^{2}=0.01 & - \\
\mathrm{F}_{(5,3227)}=1.4, \eta^{2}=0 & \mathrm{~F}_{(4,1601)}=2.4^{*}, \eta^{2}=0.01 \\
\mathrm{~F}_{(1,3227)}=0.28, \eta^{2}=0 & \mathrm{~F}_{(1,1601)}=0.1, \eta^{2}=0 \\
\mathrm{~F}_{(1,3227)}=4.8^{*}, \eta^{2}=0 & \mathrm{~F}_{(1,1601)}=5.0^{*}, \eta^{2}=0 \\
\mathrm{~F}_{(1,3227)}=2.1^{*}, \eta^{2}=0 & - \\
\mathrm{F}_{(24,3227)}=51.1^{* *}, \eta^{2}=0.38 & \mathrm{~F}_{(24,1601)}=19.4^{* *}, \eta^{2}=0.29
\end{array}
$$

\section{Discussion}

Results indicate that sleep deprivation and time on task cause a general decrement in cortical responsiveness to incoming visual stimuli while driving. Lambda responses were lower in amplitude during night driving and with increasing time on task, whereas levels of subjective sleepiness and line crossings were higher under both conditions. Furthermore, smaller lambda responses were associated with higher subjective sleepiness and more line crossings.

The present study indicates that the lambda response is sensitive to night driving, which includes sleep deprivation, exposure to the circadian low, and time on task. However, the effect size was small. This result is in line with those of earlier studies using externally triggered ERPs (Gosselin et al., 2005, Hoedlmoser et al., 2011). Our results suggest that sleep deprivation and time on task cause a general decrement in cortical responsiveness to incoming visual stimuli (Chee, 2015, Boonstra et al., 2005), as reflected by lower lambda responses (Takeda et al., 2016, Takeda et al., 2012, Yagi, 1981, Jagla et al., 2007). Therefore, the lambda response may not be influenced only by exogenous factors (Ries et al., 2018), but also by active visual information processing. Note that there is no significant interaction between sleep deprivation and time on task for the lambda response as there is for subjective sleepiness and line crossings. This may indicate that the lambda response represents sleepiness in a different way from these other measures.

The analyses also suggest that the lambda responses could predict line crossings, a widely used indicator of the effect of driver sleepiness. However, the usefulness of EFRPs in this context requires improved model fit and reduced residuals. A linear mixed-effects model was chosen here, but it is possible that a nonlinear model would improve the model fit between the lambda response and KSS/line crossings. One also needs to determine the ideal trigger variable for the EFRP, as both saccade detection and saccade size measurement may perform better with eye trackers than EOG, in which it is difficult to pinpoint the exact times of fixation onset. Another issue concerns the considerable individual differences. Inter- and intra-individual differences often influence psychophysiological measures of driver sleepiness (Lal and Craig, 2001), and unfortunately the lambda response is no exception. 
The lambda response is internally generated, continuous, and sensitive to sleepiness, which should make it useful as an indicator of sleepiness in laboratory research contexts. Here, a driving simulator was used, which provides a well-controlled environmental context in which we manipulated light condition and the level of visual stimulation. The next step is to test the sensitivity of lambda responses while varying environmental stimulation in short intervals to evaluate the acute effects of external stimulation. Further studies should also investigate the sensitivity of lambda responses in reallife driving.

Finally, the analyses also corroborate earlier studies showing an increase in KSS levels and line crossings during night driving and with time on task (Anund et al., 2008, Hallvig et al., 2013, Philip et al., 1999). The peak levels reached at the end of the night drive were very high, with medium to high effect sizes for night driving plus time on task. The results support the notion that KSS ratings are very sensitive to sleepiness-inducing factors (Åkerstedt et al., 2014) and predict line crossings (Hallvig et al., 2014, Åkerstedt et al., 2013). For line crossings, the effect size was small to medium, but clearly significant.

Ever since eye movement-related potentials were introduced by Gaarder et al. (1964), it has been discussed whether these ERPs are true brain responses or artefacts caused by oculomotor activity. In this study, it is therefore relevant to question whether the lambda response really is a sign of reduced cortical processing or whether it is just a proxy measurement for eye movement behaviour that varies across different levels of driver sleepiness. To reduce the likelihood of the latter, fixation onsets cooccurring with eye blinks were excluded from the ensemble averages. The number of fixations and the amplitude of the preceding saccade were also included as factors in the analyses. However, neither of these countermeasures will rule out the possibility that the reduced lambda response is a by-product of eye fatigue. More controlled laboratory experiments are needed to investigate this further.

In summary, the lambda response decreased due to night driving and time on task, while KSS and number of line crossings increased. The lambda response also predicted line crossings and KSS. It is suggested that the lambda response can be used to investigate cognitive impairment induced by sleep deprivation while driving and that, after further refinement, it may be useful as an objective measure of sleepiness.

\section{Acknowledgements}

The authors would like to acknowledge VINNOVA for supporting the Vehicle Driver Monitoring research project.

\section{References}

Ahlstrom, C., Jansson, S. and Anund, A. Local changes in the wake electroencephalogram precedes lane departures. Journal of sleep research, 2017, 26: 816-19. 
Ahlström, C., Anund, A., Fors, C. and Åkerstedt, T. The effect of daylight versus darkness on driver sleepiness: a driving simulator study. Journal of sleep research, 2018a, 27: e12642.

Ahlström, C., Anund, A., Fors, C. and Åkerstedt, T. Effects of the road environment on the development of driver sleepiness in young male drivers. Accident Analysis \& Prevention, 2018b, 112: 127-34.

Anund, A., Kecklund, G., Vadeby, A., Hjälmdahl, M. and Åkerstedt, T. The alerting effect of hitting a rumble strip--a simulator study with sleepy drivers. Accident Analysis and Prevention, 2008, 40: 1970-76.

Berg, P. and Davies, M. B. Eyeblink-related potentials. Electroencephalography and Clinical Neurophysiology, 1988, 69: 1-5.

Boonstra, T., Daffertshofer, A. and Beek, P. Effects of sleep deprivation on event-related fields and alpha activity during rhythmic force production. Neuroscience letters, 2005, 388: 27-32.

Boonstra, T., Stins, J., Daffertshofer, A. and Beek, P. Effects of sleep deprivation on neural functioning: an integrative review. Cellular and molecular life sciences, 2007, 64: 934.

Bulling, A., Ward, J. A., Gellersen, H. and Troster, G. Eye movement analysis for activity recognition using electrooculography. IEEE transactions on pattern analysis and machine intelligence, 2011, 33: 741-53.

Chee, M. W. Limitations on visual information processing in the sleep-deprived brain and their underlying mechanisms. Current opinion in behavioral sciences, 2015, 1: 56-63.

Daly, I., Scherer, R., Billinger, M. and Müller-Putz, G. FORCe: Fully online and automated artifact removal for brain-computer interfacing. IEEE transactions on neural systems and rehabilitation engineering, 2015, 23: 725-36.

Filtness, A. J., Reyner, L. A. and Horne, J. A. Driver sleepiness-comparisons between young and older men during a monotonous afternoon simulated drive. Biological Psychology, 2012, 89: 58083.

Gaarder, K., Krauskopf, J., Graf, V., Kropfl, W. and Armington, J. C. Averaged brain activity following saccadic eye movement. Science, 1964, 146: 1481-83.

Gosselin, A., De Koninck, J. and Campbell, K. B. Total sleep deprivation and novelty processing: implications for frontal lobe functioning. Clinical Neurophysiology, 2005, 116: 211-22.

Hallvig, D., Anund, A., Fors, C., Kecklund, G. and Akerstedt, T. Real driving at night - Predicting lane departures from physiological and subjective sleepiness. Biological Psychology, 2014, 101: 18-23.

Hallvig, D., Anund, A., Fors, C. et al. Sleepy driving on the real road and in the simulator--A comparison. Accident Analysis and Prevention, 2013, 50: 44-50.

Hoedlmoser, K., Griessenberger, H., Fellinger, R. et al. Event-related activity and phase locking during a psychomotor vigilance task over the course of sleep deprivation. Journal of sleep research, 2011, 20: 377-85.

Jagla, F., Jergelova, M. and Riečanský, I. Saccadic eye movement related potentials. Physiological research, 2007, 56

Jammes, B., Sharabty, H. and Esteve, D. Automatic EOG analysis: A first step toward automatic drowsiness scoring during wake-sleep transitions Somnologie - Schlafforschung und Schlafmedizin, 2008, 12: 227-32.

Kazai, K. and Yagi, A. Comparison between the lambda response of eye-fixation-related potentials and the P100 component of pattern-reversal visual evoked potentials. Cognitive, Affective, \& Behavioral Neuroscience, 2003, 3: 46-56.

Krause, A. J., Simon, E. B., Mander, B. A. et al. The sleep-deprived human brain. Nature Reviews Neuroscience, 2017, 18: 404.

Lal, S. K. and Craig, A. A critical review of the psychophysiology of driver fatigue. Biological Psychology, 2001, 55: 173-94.

Luck, S. J., Woodman, G. F. and Vogel, E. K. Event-related potential studies of attention. Trends in cognitive sciences, 2000, 4: 432-40. 
Nikolaev, A. R., Meghanathan, R. N. and Van Leeuwen, C. Combining EEG and eye movement recording in free viewing: Pitfalls and possibilities. Brain and cognition, 2016, 107: 55-83.

Nir, Y., Andrillon, T., Marmelshtein, A. et al. Selective neuronal lapses precede human cognitive lapses following sleep deprivation. Nature medicine, 2017, 23: 1474.

Philip, P., Taillard, J., Quera-Salva, M., Bioulac, B. and Åkerstedt, T. Simple reaction time, duration of driving and sleep deprivation in young versus old automobile drivers. Journal of sleep research, 1999, 8: 9-14.

Quercia, A., Zappasodi, F., Committeri, G. and Ferrara, M. Local Use-Dependent Sleep in Wakefulness Links Performance Errors to Learning. Frontiers in human neuroscience, 2018, 12: 122.

Railo, H., Koivisto, M. and Revonsuo, A. Tracking the processes behind conscious perception: a review of event-related potential correlates of visual consciousness. Consciousness and cognition, 2011, 20: 972-83.

Rennie, C. J., Robinson, P. A. and Wright, J. J. Unified neurophysical model of EEG spectra and evoked potentials. Biological cybernetics, 2002, 86: 457-71.

Ries, A. J., Slayback, D. and Touryan, J. The fixation-related lambda response: Effects of saccade magnitude, spatial frequency, and ocular artifact removal. International Journal of Psychophysiology, 2018, 134: 1-8.

Ries, A. J., Touryan, J., Ahrens, B. and Connolly, P. The impact of task demands on fixation-related brain potentials during guided search. PloS one, 2016, 11: e0157260.

Solís-Marcos, I., Galvao-Carmona, A. and Kircher, K. Reduced attention allocation during short periods of partially automated driving: an event-related potentials study. Frontiers in human neuroscience, 2017, 11: 537.

Strayer, D. L., Turrill, J., Cooper, J. M., Coleman, J. R., Medeiros-Ward, N. and Biondi, F. Assessing cognitive distraction in the automobile. Human Factors, 2015, 57: 1300-24.

Takeda, Y., Sato, T., Kimura, K., Komine, H., Akamatsu, M. and Sato, J. Electrophysiological evaluation of attention in drivers and passengers: Toward an understanding of drivers' attentional state in autonomous vehicles. Transportation Research Part F: Traffic Psychology and Behaviour, 2016, 42: 140-50.

Takeda, Y., Yoshitsugu, N., Itoh, K. and Kanamori, N. Assessment of attentional workload while driving by eye-fixation-related potentials. Kansei Engineering International Journal, 2012, 11: 121-26.

Van Dinteren, R., Arns, M., Jongsma, M. L. and Kessels, R. P. P300 development across the lifespan: a systematic review and meta-analysis. PloS one, 2014, 9: e87347.

Wascher, E., Heppner, H. and Hoffmann, S. Towards the measurement of event-related EEG activity in real-life working environments. International Journal of Psychophysiology, 2014, 91: 3-9.

Wiberg, H., Nilsson, E., Lindén, P., Svanberg, B. and Poom, L. Physiological responses related to moderate mental load during car driving in field conditions. Biological Psychology, 2015, 108 : $115-25$.

Yagi, A. Saccade size and lambda complex in man. Physiological Psychology, 1979, 7: 370-76.

Yagi, A. Visual signal detection and lambda responses. Electroencephalography and Clinical Neurophysiology, 1981, 52: 604-10.

Åkerstedt, T., Anund, A., Axelsson, J. and Kecklund, G. Subjective sleepiness is a sensitive indicator of insufficient sleep and impaired waking function. Journal of sleep research, 2014, 23: 240-52.

Åkerstedt, T., Hallvig, D., Anund, A., Fors, C., Schwarz, J. and Kecklund, G. Having to stop driving at night because of dangerous sleepiness - awareness, physiology and behaviour. Journal of sleep research, 2013, 22: 380-88. 